A new technique is described for the measurement of vibrations in the temporal bones of an isolated middle ear. The precise recording of vibrations in the middle ear is of importance for the construction and improvement of a middle ear prosthesis. ${ }^{1}$ The method of measurement is based on a transformation of mechanical vibrations into magnetic flux variations. This is performed by attaching a small piece of permanent magnetic material to the eardrum or middle ear ossicles. The magnetic flux variations caused by vibrations of the eardrum or ossicles during application of sound can be measured by means of a SQUID magnetometer.

\title{
The use of a SQUID magnetometer for middle ear research
}

\author{
W.L.C. Rutten, M.J. Peters, C.J. Brenkman, H. Mol, J.J. Grote and \\ L.C. van der Marel
}

Key words: cryogenic, SQUID, magnetometer, ear

\section{Introduction}

For the construction and improvement of a 'total alloplastic middle ear', 1 it is necessary to measure transfer properties of the prosthesis. One should know amplitude and phase characteristics at the input and output of the system, the tympanic membrane and stapes footplate respectively. Furthermore, knowledge of the position and direction of rotation axes in the ossicular system in the human temporal bone seems to be of interest, in order to construct an equivalent of the normal middle ear transmission. The measuring technique should meet the following demands: it must leave the structure and proper functioning of the ear (or the implant) intact, the middle ear being loaded by the intact cochlea; the technique must be sensitive enough to record vibration amplitudes of the order of $10^{-10} \mathrm{~m}$; and if possible, it should be applicable to vibratory measurements on in vivo implanted prostheses. Already existing sophisticated methods such as the capacitive probe method, ${ }^{2}$ laser interferometry, ${ }^{4}$ holography ${ }^{5}$ or the Mössbauer technique ${ }^{3}$ only meet one or two of these demands. The main disadvantage of the first three methods is that invasive surgical technical techniques are needed, either to position the capacitive probe into the middle ear, or to obtain a free optical reflection path. Furthermore, these methods provide information about vibrations in one direction only, unless the probe is repositioned or a different light path is created. Thus, it is not easy to study vectorial properties of vibrations. The Mössbauer method does not allow measurements in vivo, due to the use of a radioactive source.

A new method was developed and tested, making use of a SQUID magnetometer. Using this method, mechanical

WLCR, CJB, JJG are from the ENT Department (KNO),

University Hospital, Leiden, The Netherlands. MJP, HM, LGM are

from Twente University of Technology, Enschede, The Netherlands.

Paper received 4 February 1982. vibrations are transformed into magnetic flux variations by glueing a small magnet to the middle ear structure under study. This offers several advantages. For example, positioning of the magnet on the stapes footplate is simple. It only demands the drilling of a small hole to enter the middle ear cavity, which can be closed after positioning. In this way it is possible to measure in a closed middle ear cavity. Moreover, it will be easy to incorporate a magnet in a prosthesis, allowing measurements 'in vivo'. Another advantage is that the magnetic flux variations can be registered at a distance of several centimeters between magnet and SQUID set-up, in almost every spatial direction. Because of this 'spatial freedom', vectorial properties of vibrations can be, in principle, easily studied.

The main purpose of the investigations reported on in this article was the establishment of the applicability and sensitivity of the method. To achieve this some tympanic membrane vibratory experiments were performed on human cadaver temporal bones with intact middle ear, loaded by the intact cochlea. A calibration method of SQUID output voltage against vibration amplitude was developed as well. Calibration has been performed by a forced vibration (with known amplitude) of the magnet. The vibration exciter employed thus acts as an 'artificial ear'.

\section{Set-up and measuring procedure}

Measurements were performed in a non-soundproof hardwalled room which was not magnetically shielded. Freefield pure-tone sinusoidal acoustic stimulation was used with the loudspeaker positioned about $3 \mathrm{~m}$ from the SQUID magnetometer. Sound pressure levels were measured at the site of the eardrum by a carefully calibrated (at discrete frequencies) probe-tube microphone. Stimulatory frequencies equalled calibration frequencies (of the microphone) within $1 \mathrm{~Hz}$. The SQUID magnetometer set-up consisted of 
a SQUID sensor, connected to a symmetrical second order flux transformer, with a baseline of $9 \mathrm{~cm}$ and a diameter of $3 \mathrm{~cm} .^{6}$ This assembly was immersed in liquid helium contained in a cryostat. The magnet was a small cube of Samarium Cobalt $\mathrm{V}$, edge dimension $1.2 \mathrm{~mm}$, weight $16 \mathrm{mg}$, which was glued on the eardrum of an isolated temporal bone. During the two days between death and the start of the measurements the temporal bone was stored in a physiological salt solution. The external ear canal was removed, leaving $1 \mathrm{~mm}$ above the fibrous anulus of the eardrum intact. The prepared temporal bone was encapsulated in a rectangular block of modelling clay the eardrum lying in the upper plane. The distance between the sample and plane of the bottom turn of the gradiometer was $2.2 \mathrm{~cm}$. The SQUID output was led via an RF head (SHE $300 \mathrm{RF}$ head) to an electronic processing unit (SHE 330 control unit). Amplitude of the resulting sinusoidal output was detected by a lock-in amplifier (PAR-129) and voltmeter (Bruèl and Kjear type 2425).
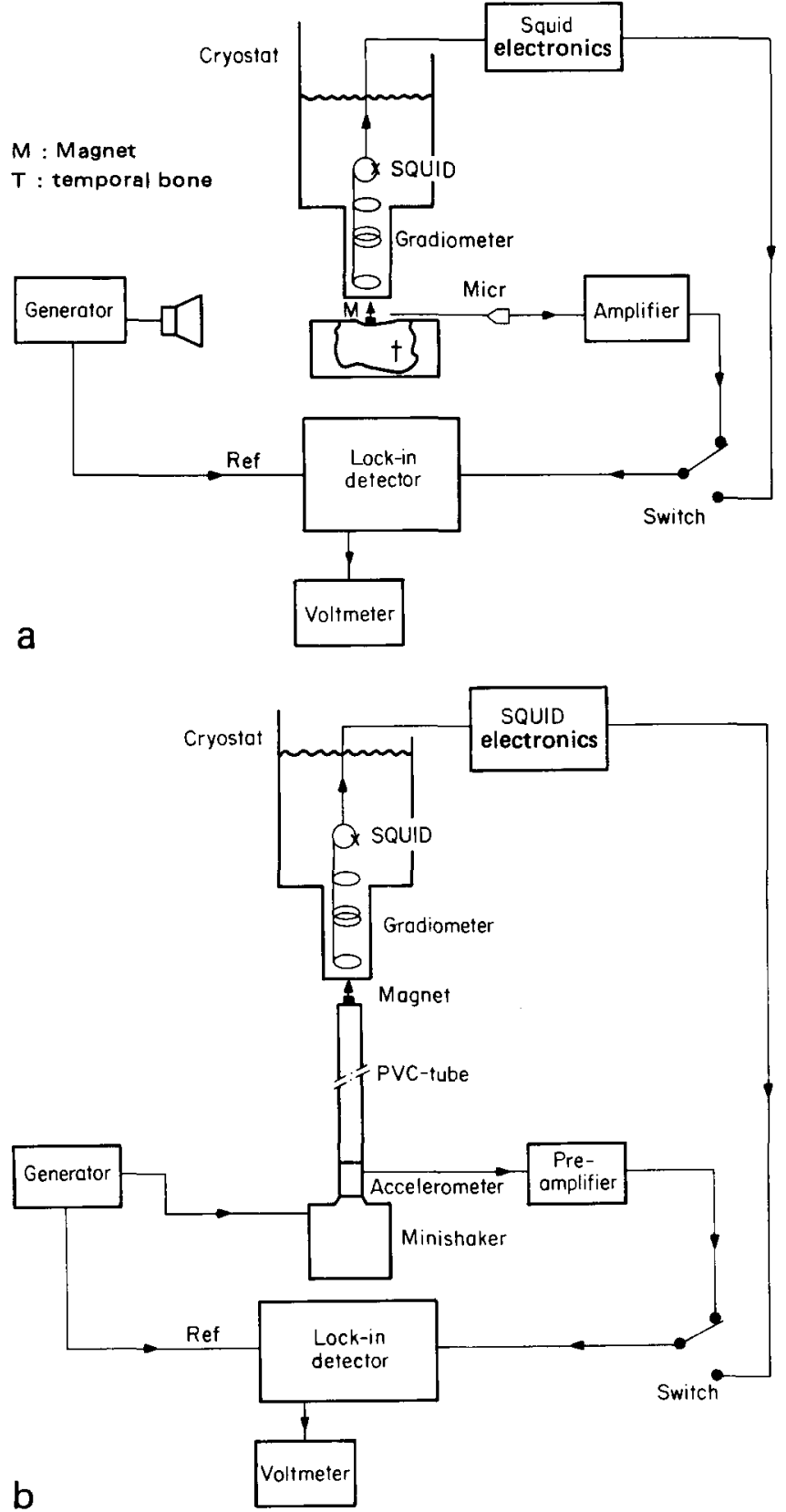

Fig. 1 a - Set-up for magnetic detection of eardrum vibrations in isolated human middle ears; $b$ - Calibration set-up, using a vibration exciter as 'artificial ear'
In order to calibrate the output voltage $V$ (linearly related to the magnetic flux changes in the flux transformer) against vibration amplitude, the sample was mounted on top of a rigid PVC tube (length $47 \mathrm{~cm}$, diameter $1.8 \mathrm{~cm}$ ). The tube was firmly connected to a calibrated accelerometer (Bruèl and Kjaer impedance head type 8000). This assembly of accelerometer, tube and sample was then brought into sinusoidal vibration by a vibration exciter (B and $\mathrm{K}$ minishaker type 4810). Accelerometer output voltages were converted into displacement voltages by B and K type 4292 and led to a PAR 129 lock-in amplifier. In this way a calibration factor in $10^{-10} \mathrm{~m} \mathrm{mV}^{-1}$ was obtained as a function of the distance between pick-up loop and magnet. The calibration factor was frequency independent except for $800 \mathrm{~Hz}$ and odd harmonics. This was due to resonance effects in the PVC tube. The noise caused by stray fields from the minishaker was only a few percent of the signal measured. This was checked by repeating the calibration procedure without the magnet present. A sketch of the two set-ups is given in Figs 1a and $1 b$.

\section{Theory}

The small magnet is considered as a magnetic dipole with magnetic dipole moment $\mathrm{m}$. Our sample has a magnetic dipole strength of $m=12 \times 10^{-4} \mathrm{Am}^{2}$. This figure is established experimentally and is in accordance with the manufacturer's specifications.

The relative position of the magnet fixed to the eardrum is schematically given in Fig. 2. The magnetic dipole is placed on the axis of the flux transformer and points in the direction of the unit vector of the plane of the loops. A polar coordinate system is chosen with the position of the sample as the origin and $e_{z}=m /|m|$. The position of a point of a loop is specified by $\mathbf{r}=(\rho, \alpha, z)$.

The magnetic scalar potential caused by a dipole $\mathrm{m}$ is:

$$
U=\mu_{0} \frac{\mathbf{m} \cdot \mathbf{r}}{4 \pi r^{3}}
$$

The $z$-component of the magnetic induction is

$$
B_{\mathrm{z}}=-\frac{\partial U}{\partial z}=-\frac{\mu_{0} m}{4 \pi}\left(\frac{1}{r^{3}}-\frac{3 z^{2}}{r^{5}}\right)
$$

A loop of the flux transformer has a radius $R$. The centre of loop $i$ is given by $\left(0,0, z_{\mathfrak{j}}\right)$. The flux through that loop is:

$$
\phi_{\mathrm{i}}=\int_{0}^{R} \int_{0}^{2 \pi} B_{\mathrm{z}_{\mathrm{i}}} \rho \mathrm{d} \rho \mathrm{d} \alpha
$$

Inserting $B_{\mathrm{z}}$ from (2), taking $r=\left(\rho^{2}+z^{2}\right)^{1 / 2}$ gives:

$$
\phi_{\mathrm{i}}=\frac{\mu_{0 \mathrm{~m}}}{2}\left(\frac{R^{2}}{\left(R^{2}+z_{\mathrm{i}}^{2}\right)^{3 / 2}}\right)
$$

A vibration of the dipole in the $z$-direction with amplitude $\Delta z$, where $\Delta z \ll z_{\mathrm{i}}$ causes a flux change through loop $i(i=1,2,3)$ :

$$
\Delta \phi_{\mathrm{i}} \approx \frac{\partial \phi}{\partial z} \Delta z=-\frac{3}{2} \frac{\mu_{0} m R^{2} z_{\mathrm{i}}}{\left(R^{2}+z_{\mathrm{i}}{ }^{2}\right)^{5 / 2}} \Delta z
$$

The total flux in the flux transformer is:

$$
\Delta \phi=\Delta \phi_{1}-2 \Delta \phi_{2}+\Delta \phi_{3}
$$




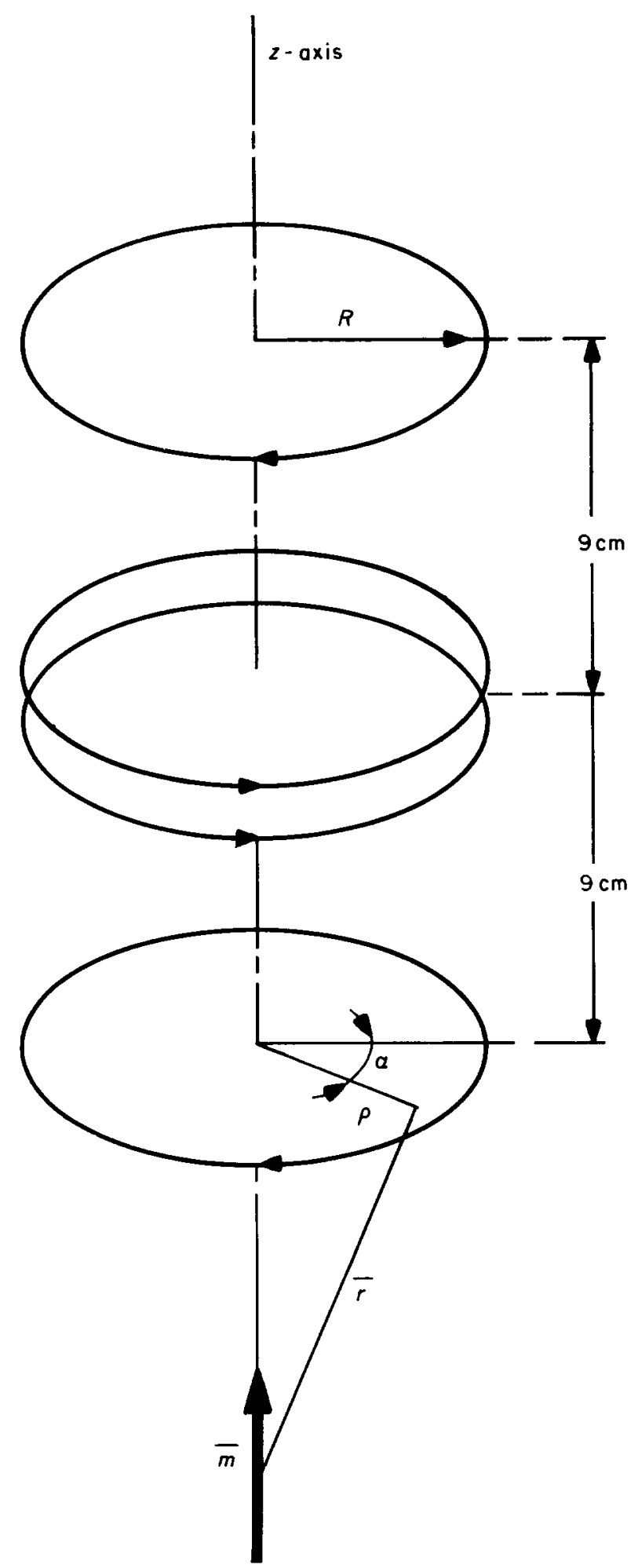

Fig. 2 The relative position of the magnetic dipole fixed to the eardrum with respect to the flux transformer

Inserting (5) into (6) gives for $\Delta z=10^{-10} \mathrm{~m}, z_{1}=2.2 \mathrm{~cm}$, $z_{2}=11.2 \mathrm{~cm}, z_{3}=20.2 \mathrm{~cm}$ and $R=1.5 \mathrm{~cm}$ a flux change of $8.4 \times 10^{-15} \mathrm{~Wb} \approx 4 \phi_{0}$, where $\phi_{0}$ is the flux quantum. The contributions through the loops 2 and 3 can be ignored.

From (2) follows for a small vibration in a direction perpendicular to the $z$-direction:

$$
\Delta B_{z} \approx \frac{\partial B_{z}}{\partial x} \Delta x+\frac{\partial^{2} B_{z}}{\partial x^{2}} \frac{(\Delta x)^{2}}{2}+\ldots
$$

The first order term is:

$$
\frac{\partial B_{\mathrm{z}}}{\partial x} \Delta x=-\frac{\mu_{0} m}{4 \pi}\left(-\frac{3}{2} \frac{2 x}{r^{5}}-\frac{15 z^{2} x}{r^{7}}\right) \Delta x
$$

Inserting this into (3), with $r^{2}=\rho^{2}+z^{2}$ and $x=\rho \cos \alpha$ gives, after integration, a first order contribution to the flux change of zero. The second order term gives a nonzero contribution. Inserting the values as mentioned above shows that $\Delta \phi \approx 2 \times 10^{-28} \mathrm{~Wb}$; so small vibrations in a direction perpendicular to the axis are not seen.

When the dipole changes its direction as well, for example when it rotates over $10^{-8} \mathrm{rad}$, which corresponds with a displacement of $10^{-10} \mathrm{~m}$ when the centre of the rotation is at $1 \mathrm{~cm}$ from the sample, this yields flux changes which can also be neglected. The results of such calculations are presented elsewhere. ${ }^{7}$ From calculations given in Reference 7 , it follows that directly above the centre of rotation at a distance of $4 \mathrm{~cm}$, the flux change $=3 \times 10^{-13} \mathrm{~Wb}$ for a magnetic moment of $1 \mathrm{Am}^{2}$ and for a small angular displacement of $\Delta \alpha=0.15 \times 10^{-3} \mathrm{rad}$. In this situation $\Delta \phi$ is linearly proportional to $(\Delta \alpha)^{2}$ because the first term in the Taylor's expansion is zero. This means that in our case we would find at $4 \mathrm{~cm}$ distance a flux change of $2 \times 10^{-24} \mathrm{~Wb}$. When the distance is $2.2 \mathrm{~cm}$, the order of the flux change will be such that small rotations are not seen.

Looking at the theoretical results we can draw the following conclusions: When the flux transformer is directly above the dipole, with the axis and the dipole pointing in the same direction, the sensitivity of a SQUID magnetometer is enough to detect vibrations of $10^{-10} \mathrm{~m}$ by means of a very small magnet. SQUIDS which are for sale have a sensitivity better than $10^{-3} \phi_{0}(\mathrm{~Hz})^{-1 / 2}$ and the flux reduction factor of a flux transformer is in the order of $10^{-2}$. The applied sound has a known frequency. The response has the same frequency. This makes it possible to enhance the sensitivity of the magnetometer using a lock-in amplifier.

Vibrations in a direction perpendicular to the axis cannot be registered nor can small rotations.

Because $\Delta z \ll z_{1}$ and because the upper loops of the flux transformer play no role, the flux change ie the output voltage of the magnetometer is linearly proportional to the displacement and is given by (5). From (5) it follows that the sensitivity of the system is enhanced when the radius of the pick-up loop $R$ and the distance of that loop to the magnet are diminished. So a pick-up loop with a small radius and more turns will be favourable.

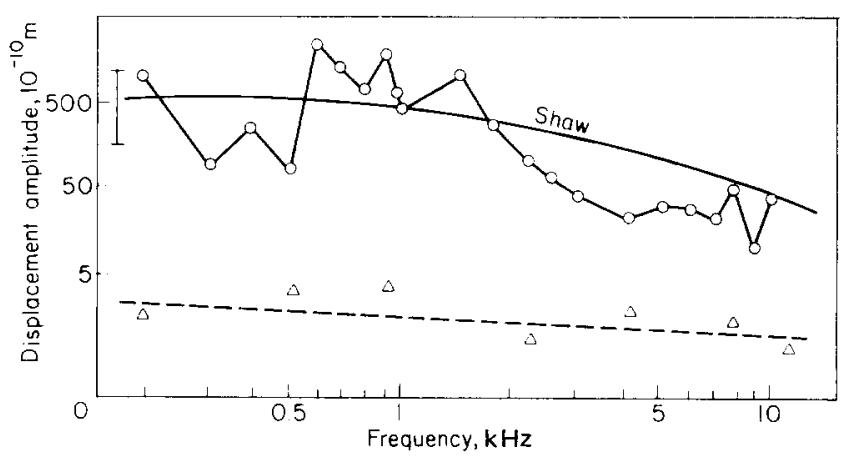

Fig. 3 Eardrum displacement amplitude versus frequency, in response to a constant input sound level of $90 \mathrm{~dB}$ SPL. - experimental data; - data of Shaw, ie an average of data given by six authors. - - minimal detectable displacement amplitude. The vertical bar at the left indicates the variability across the six author's data 
For measurements in vivo the distance between the eardrum and pick-up loop will be too large due to the ear canal. A flux transformer at room temperature, which does not give too high losses, has to be constructed. Calculations and tentative experiments showed that it is possible.

The alignment of the direction of the dipole along the axis of the flux transformer can be achieved with the help of a flux gate magnetometer. The SQUID magnetometer is not suitable for this purpose because with this instrument only changes in the flux are detected.

\section{Experimental results}

For calibration the vibratory amplitude of the minishaker was held constant at $25.5 \times 10^{-10} \mathrm{~m}$. With a distance of $2.2 \mathrm{~cm}$ between sample and gradiometer and for the $16 \mathrm{mg}$ sample a calculation factor was obtained of $59.3 \times 10^{-10} \mathrm{~m} \mathrm{mV}^{-1}$. Except for the resonance frequency regions of the PVC tube mentioned above, this factor was almost independent of the frequency in the region between 200 and $10000 \mathrm{~Hz}$ (the SQUID electronics response characteristic is flat within +4 and $-6 \mathrm{~dB}$ up to $10000 \mathrm{~Hz}$ ). The noise level actually measured at the detector output had a typical RMS value (at $1000 \mathrm{~Hz}$ ) of $25 \mu \mathrm{V}$. This implies a minimal detectable RMS amplitude of about $3 \times 10^{-10} \mathrm{~m}$ (for a signal-to-noise ratio of 2). The ambient noise level (due to fluctuations in the geomagnetic field and local field sources such as lifts, motors) may vary by a factor of four within several days.

The magnet was glued with histo-acrylate on the eardrum. The distance between sample and pick-up loop of the gradiometer was $2.2 \mathrm{~cm}$. A frequency response curve for the vibration peak amplitude $\Delta z$ was measured.

It is well known that middle ear vibration amplitudes are linearly proportional to the pressure level of the sound input, up to about $120 \mathrm{~dB}$ SPL. ${ }^{8}$ (In acoustics SPL means sound pressure level. The $0 \mathrm{~dB}$ SPL reference level is chosen such that $0 \mathrm{~dB}$ SPL corresponds to a RMS sound pressure of $2 \times 10^{-5} \mathrm{Nm}^{-2}$ ). We used this proportionality as a check on our measurements at several frequencies and indeed it was observed (results not shown).

Fig. 3 (open circles) shows a frequency response curve for the eardrum in a temporal bone of a 76 year old male. Open circles denote eardrum displacement amplitudes in response to a sound level of $90 \mathrm{~dB}$ SPL. At most frequencies, data were taken at lower levels (down to $54 \mathrm{~dB} \mathrm{SPL}$ ) as well. At some frequencies, amplitude was measured for one input sound level only. In those cases, amplitude was calculated for $90 \mathrm{~dB}$ SPL input using the above mentioned linear inputoutput relationship. The dashed line in Fig. 3 indicates the minimal amplitude which could be measured, being about $1.5 \times 10^{-10} \mathrm{~m}$ at $1000 \mathrm{~Hz}$.

\section{Discussion and conclusions}

We compared our results with results from the literature. Shaw ${ }^{8}$ collected impedance data from six other studies and calculated their mean values. We converted this average impedance data to eardrum displacements in response to $90 \mathrm{~dB}$ SPL sound input, taking an eardrum area of $0.65 \mathrm{~cm} .^{2}$ The result is drawn in Fig. 3 as the solid line labelled 'Shaw'. The vertical bar at the left indicates the variability of the mean. (In general such rather large variability is not uncommon in biological systems.) Our results compare favourably well with Shaw's data, the variability taken into account, although between 200 and $600 \mathrm{~Hz}$ displacement values are very low. Immediate remeasurement of the whole curve yielded results, which were identical to the former ones within a few percent.

We conclude that the SQUID magnetometer method has proved to be a simple and sensitive method for measuring in vitro very small vibration amplitudes in human temporal bones.

Results obtained for eardrum vibration amplitudes agree well with findings in the literature.

The authors would like to thank Mrs J J Wevers-Henke and J A Ulfman for their helpful discussions. We also gratefully acknowledge technical assistance in Leiden as well as in Enschede.

\section{References}

Grote, J.J., Kuijpers, W. Arch Otolaryngol 106 (1980) 560 Wilson, J.P., Johnstone, J.R. JASA 57 (1975) 705 Gilad, P. et al JASA 41 (1967) 1232 Tonndorf, J., Khanna, S.M. JASA 44 (1968) 1546 Khanna, S.M. Tonndorf, J. JASA 51 (1972) 1904 Peters, M.J. thesis Twente University of Technology, The Netherlands (1981)

7 Peters, M.J. Dunajski, Z., Meyssen, T.E.M., Breukink, E.W., Wevers-Henke, J.J. submitted to Cryogenics

8 Shaw, E.A.G. in Handbook of Sensor Physiology V/1, eds. W.D. Keidel and W.D. Neff. Springer Verlag, Berlin, Heidelberg, New York 